\title{
Respiratory chain complex III deficiency in patients with tRNA-leu mutation
}

\author{
J. Jiang ${ }^{1}$, X.L. Wang ${ }^{2}$ and Y.Y. Ma $^{3,4}$ \\ 1'Department of Oncology, Affiliated Hospital of Qinghai University, Xining, China \\ 2Operation Room, Affiliated Hospital of Qinghai University, Xining, China \\ ${ }^{3}$ Department of Pediatrics, Chinese Liberation Army General Hospital, \\ Beijing, China \\ ${ }^{4}$ Department of Pediatrics, Affiliated Hospital of Qinghai University, Xining, China \\ Corresponding author: Y.Y. Ma \\ E-mail: xnmayanyan@126.com
}

Genet. Mol. Res. 14 (4): 18629-18636 (2015)

Received August 25, 2015

Accepted October 25, 2015

Published December 28, 2015

DOI http://dx.doi.org/10.4238/2015.December.28.12

\begin{abstract}
The aim of this study was to investigate the clinical and genetic profiles of mitochondrial disease resulting from deficiencies in the respiratory chain complex III. Three patients, aged between 8 months and 12 years, were recruited for this study. The activities of mitochondrial respiratory chain complexes in the peripheral leucocytes were spectrophotometrically measured. The entire mitochondrial DNA (mtDNA) sequence was analyzed. Samples obtained from the three patients and their families were subjected to restriction fragment length polymorphism and gene sequencing analyses. mtDNA copy numbers of all patients and their mothers were analyzed. The patients displayed nervous system impairment, including motor and mental developmental delay, hypotonia, and motor regression. Two patients also suffered from Leigh syndrome. Assay of the mitochondrial respiratory chain enzymes revealed an isolated complex III deficiency in the three patients. The m.3243 A>G mutation was detected in all patients and their mothers. The mutation loads were $48.3,57.2$, and $45.5 \%$ in the patients, and $20.5,16.4$, and $23.6 \%$ in their respective mothers. The leukocyte mtDNA copy numbers of the patients
\end{abstract}


and their mothers were within the control range. The clinical manifestation and genetics were observed to be very heterogeneous. Patient carrying an m.3243 $A>G$ mutation may biochemically display a deficiency in the mitochondrial respiratory chain complex III.

Key words: Respiratory chain complex III deficiency; Mitochondrial DNA; 3243A>G mutation

\section{INTRODUCTION}

The mitochondrial respiratory chain is composed of five enzyme complexes, which produce energy through intracellular electron transfer and oxidative phosphorylation. All enzyme complexes, excluding complex II, are encoded by nuclear and mitochondrial DNA. Complex III, also termed Cytochrome c reductase (or bc1 complex), is located in the middle of the mitochondrial respiratory chain. It consists of ten nuclear-encoded subunits and one mitochondrial-encoded subunit; three of these subunits are involved in electron transfer: Rieske iron sulfur protein, cytochrome b, and cytochrome c1. Complex III catalyzes the transfer of electrons from complex I (NADH dehydrogenase) and II (succinate dehydrogenase) to cytochrome $\mathrm{C}$, which utilizes the released energy for vectorial translocation of two protons from the matrix to the intermembrane (Iwata et al., 1998). Previous studies have indicated complex III to be the primary site of ROS production during hypoxia. The suppression of the expression of the Rieske iron-sulfur protein (of complex III) allows for the stabilization of hypoxia-induced HIF-1 alpha to be attenuated, and a decrease in ROS production. This indicated that the mitochondria function as $\mathrm{O}_{2}$ sensors, signaling for hypoxic $\mathrm{HIF}-1$ alpha and HIF-2 alpha stabilization by releasing the ROS to the cytosol (Guzy et al., 2005).

Mitochondrial respiratory chain disorders are degenerative diseases with a clinically heterogeneous manifestation, occurring in approximately 1:5000-8000 of all live-born infants (Schafer et al., 2004). Despite the wide clinical phenotype of multi-organ lesions, tissues with high energy requirements, such as the muscle, heart, and brain tissues, are particularly vulnerable. Defects in complexes I and IV, resulting from mutations in the mitochondrial or nuclear genome, account for a majority of the cases of mitochondrial disease; a wide spectrum of the biochemical data and clinical presentations of such defects are well-known. On the other hand, isolated complex III deficiencies are relatively rare, and very little is known about the occurrence and characteristics of complex III deficiency in the mitochondrial respiratory chain in China. In this paper, we have described three patients with complex III deficiencies, who displayed varying clinical manifestations of the disease.

\section{MATERIAL AND METHODS}

\section{Patients}

Three patients, aged between 8 months and 12 years, were recruited for this study. The occurrence of typical aminoacidopathies, organic acidurias, and mitochondrial $\beta$-oxidation defects were excluded by blood amino acid, acylcarnitine, and urinary organic acid analyses, respectively, prior to conducting tests for mitochondrial disorders. Informed consent was obtained from the parents of all patients included in the study. This study was approved by the ethics committee of 
the Chinese Liberation Army General Hospital.

\section{Methods}

\section{Assays for mitochondrial respiratory chain complexes I-V}

The peripheral leukocytes obtained from the patients were subjected to spectrophotometric assays specific for mitochondrial respiratory chain complexes. The specific activity of complex I was measured at $340 \mathrm{~nm}$ based on the decrease in absorbance caused by NADH oxidation at 340 $\mathrm{nm}$. Rotenone $(2 \mu \mathrm{g} / \mathrm{mL})$ was added to the mixture solution prior to the addition of the mitochondria protein in order to determine the unspecific activity of complex I, as it is the rotenone-sensitive $\mathrm{NADH}$ :ubiquinone oxidoreductase activity. Complex II specific activity was measured at $600 \mathrm{~nm}$ by following the decrease in absorbance resulting from dichlorophenol indophenol oxidation. Complex I + III specific activity was measured at $550 \mathrm{~nm}$ by following the increase in absorbance due to cytochrome c reduction. Complex IV specific activity was measured at $550 \mathrm{~nm}$ by following the decrease in absorbance due to the oxidation of reduced cytochrome c. Complex $\mathrm{V}$ was assayed in the reverse direction, as F1-ATPase. The assay relied on linking the ATPase activity to NADH oxidation through the conversion of phosphoenolpyruvate to pyruvate by $\mathrm{PK}$, and subsequently, pyruvate to lactate by LDH. Other unspecific activities contribute a significant blank rate, which can be minimized by inhibiting the $\mathrm{Ca}^{2+}$-ATPase activity suing EGTA, and determining the oligomycinsensitive activity. The activity of each complex was expressed both as the rate $(\mathrm{nmol} / \mathrm{min} / \mathrm{mg})$ of mitochondrial protein, and as the ratio of the rate of citrate synthase. The detailed method has been described in a previous report (Ma et al., 2011).

\section{Mitochondrial gene sequence analysis}

All patients were subjected to a mitochondrial gene sequence analysis. Total DNA was extracted from the blood samples using conventional salting-out protocols (Miller et al. 1988). The entire mitochondrial genome was amplified by polymerase chain reaction (PCR) into eight overlapping fragments, using sets of light- and heavy-strand oligonucleotide primers. Each fragment was purified and analyzed by direct sequencing. The sequence results were then compared with the revised Cambridge reference sequence (http://www.mitomap.org; GenBank ID: NC_012920.1).

\section{mtDNA copy number assay}

The mitochondrial DNA (mtDNA) copy number assay method previously described by Xing et al. (2008) has been utilized in this study (Xing et al., 2008). The ratio between the multi-copy mtDNA ND1 gene and the double-copy nuclear gene hemoglobin $\beta$ was determined using an Applied Biosystems 7900 Sequence Detection System (Applied Biosystems, CA, USA). The mitochondrial ND1 and nuclear hemoglobin $\beta$ genes were individually amplified using the primers ND1-F/ND1-R and Globin-F/Globin-R, respectively. The primer sequences used were as follows: ND1, forward primer (ND1-F), 5'-ATTCGATGTTGAAGCCTGAGACT-3' and reverse primer (ND1-R), 5'-TGACCCTTGGCCATAATATGATT-3'; and globin gene, forward primer (globin-F), 5'-ACCTCAAGGGCACCTTTGC-3' and reverse primer (globin-R), 
5'-AAAACATCAAGCGTCCCATAGAC-3'. All samples were analyzed in triplicate, and the average value was used for calculation of the ratio. The $\mathrm{R}^{2}$-value for each standard curve was 0.99 or higher. Standard deviations for the cycle of threshold $(\mathrm{Ct})$ value were accepted at 0.25 . Relative mtDNA copy number was calculated using a comparative Ct method, using the equation: relative copy number $(\mathrm{mtDNA} / \mathrm{nDNA})=2^{\Delta \mathrm{Ct}}\left(\Delta \mathrm{Ct}=\mathrm{Ct}_{\beta \text { - globin }}-\mathrm{Ct}_{\mathrm{ND1}}\right)$. High reproducibility was observed using this assay, with a coefficient of variance of $8 \%$ (Birch-Machin and Turnbull, 2001).

\section{RESULTS}

\section{Clinical features}

The clinical features of the three patients are summarized in Table 1. Their parents were healthy and nonconsanguineous; patients 1 and 3 also did not show any family history of neuromuscular disease. Patient 2, however, had an old brother who died because of a similar illness. This brother was born after normal pregnancy and delivery, and developed normally until 7 months of age, when motor regression was noticed. A gradual loss of all major motor skills was observed over a period of 2 years without any preceding infection. Finally, he died of multiorgan failure at the age of 3 (years). A mitochondrial defect was suspected; however, a definitive diagnosis was never reached.

All patients were born following an uneventful pregnancy; the mothers did not report alcohol, tobacco, or illicit drug use during the pregnancy. Patients 2 and 3 were delivered vaginally without any complication, while patient 1 was delivered by caesarean section at 39 weeks of gestation, because of delayed delivery. The birth weights of these patients were in the normal range, except for patient 3 , whose birth weight of $2.0 \mathrm{~kg}$ indicated intrauterine growth retardation.

Table 1. Clinical and laboratory features ofthree patients with complex III deficiency.

\begin{tabular}{|c|c|c|c|c|}
\hline Patient & 1 & 2 & 3 & Total \\
\hline Age & $8 \mathrm{~m}$ & $10 \mathrm{~m}$ & $10 y$ & \\
\hline Gender & $\mathrm{F}$ & M & $\mathrm{F}$ & $1 \mathrm{M} / 2 \mathrm{~F}$ \\
\hline Age at onset & Birth & Birth & $9 y$ & \\
\hline Birth weight $(\mathrm{kg})$ & 3.1 & 2.8 & 2.0 & 2.63 \\
\hline Family history & - & + & - & $1 / 3$ \\
\hline Motor developmental delay & + & + & - & $2 / 3$ \\
\hline Mental developmental delay & + & - & - & $1 / 3$ \\
\hline Motor regression & - & - & + & $1 / 3$ \\
\hline Hypotonia & + & + & - & $2 / 3$ \\
\hline Epilepsy & - & - & + & $1 / 3$ \\
\hline Hyperlactacidemia & + & + & + & $3 / 3$ \\
\hline High pyruvate & + & + & - & 23 \\
\hline Low free carnitine & + & + & + & $3 / 3$ \\
\hline Low arginine & + & + & + & $3 / 3$ \\
\hline Liver involvement & - & - & - & $0 / 3$ \\
\hline Renal involvement & - & - & - & $0 / 3$ \\
\hline Magnetic Resonance Imaging & Basal ganglia & Normal & Thalamus putamen & $2 / 3$ \\
\hline
\end{tabular}

$\mathrm{M}=$ male; $\mathrm{F}=$ female; $\mathrm{m}=$ months; $\mathrm{y}=$ years.

Delayed postnatal motor activity was clinically manifested in patients 1 and 2 . Patient 1 was unable to show any head control or visual contact. Patient 2 could sit unaided at 7 months of age, but could not walk with support and hold the feeding bottle. Patient 3 showed head control 5 months of 
age, and was able to sit unaided at 12 months and walk unsupported at 24 months of age. Although the birth weight of patient 3 was $2.0 \mathrm{~kg}$ (which is below the 3th percentile), she gained weight slowly over the next several months. This patient displayed motor and mental normality at 9 years of age. Progressive fatiguability and somatic developmental retardation was observed in patient $x 3$ at 9 years of age; subsequently, the patient displayed a rapid downhill course, that is, she could not walk unsupported, and displayed dysphagia and difficulties in urination. The course of the disease was progressive in all patients. A thorough neurological examination revealed that the circumference of the head was within the normal range in all patients. No obvious dysmorphic features were observed, while the external genitalia, limbs, and skin appeared normal. Deep tendon reflexes were normal with brisk knee jerk reflexes, except in patient 3. Hypotonia was observed in patients 1 and 2.

Biochemical analyses revealed normal levels of blood glucose, ketone bodies, carnitine esters, and urine organic acid; however, we observed a mild elevation in plasma lactate. The concentration of plasma lactate was increased to 5.1, 3.9, and 4.4 mM (normal range: 0.5-2.0 mM) in patients 1, 2, and 3, respectively. Hyperpyruvemia was observed in patients 1 and 2 . We also observed reduced levels of serum arginine and free carnitine in all patients. Magnetic resonance imaging (MRI) scans of the brain revealed the presence of lesions in the basal ganglia, thalamus, or putamen in all patients excluding patient 2. The mitochondrial respiratory chain enzymes were assayed to further identify the cause of the disease.

All patients were empirically treated with carnitine, coenzyme, thiamine, vitamin $\mathrm{C}$, and vitamin $\mathrm{E}$ (for the mitochondrial disease), which provided different results. Patient 1 showed improved psychomotor development, and nearly normalized lactate levels. However, the remaining patients displayed progressive deterioration, compared to the previously observed symptoms and signs.

\section{Respiratory chain complex activity}

Biochemical analysis of the peripheral blood samples revealed an isolated complex III deficiency in all patients. The data is summarized in Table 2. The complex I activities in all patients were within the control range. The $\mathrm{NADH}$ :cytochrome $\mathrm{C}$ oxidoreductase activity, reflecting the capacity of complex I + III, was diminished. The activities of complex I + III were diminished to 47.1, 41.8 , and $37.5 \%$ of the lowest control value in patients 1,2 , and 3 , respectively (normal range: 84.4 $\pm 28.5 \mathrm{nmol} / \mathrm{min} \mathrm{mg}$ mitochondrial protein).

Table 2. Activity and comparative activity of respiratory chain enzymes in peripheral blood.

\begin{tabular}{|c|c|c|c|c|c|c|c|}
\hline & \multicolumn{2}{|c|}{ Complex I } & \multicolumn{2}{|c|}{ Complex I+III } & \multirow{2}{*}{$\begin{array}{c}\begin{array}{c}\text { Proportion of the } \\
\text { lowest control value }\end{array} \\
\%\end{array}$} & \multicolumn{2}{|c|}{$3243 \mathrm{~A}>\mathrm{G}$ mutation load } \\
\hline & Activity $^{a}$ & Compared activity & Activity & Compared activity & & Patient & Mother \\
\hline Patient 1 & 56.2 & 68.3 & 13.2 & 25.5 & 47.1 & 48.3 & 20.5 \\
\hline Patient 2 & 42.6 & 53.7 & 11.7 & 18.7 & 41.8 & 57.2 & 16.4 \\
\hline Patient 3 & 35.5 & 77.2 & 10.5 & 22.3 & 37.50 & 45.5 & 23.6 \\
\hline Control $^{c}$ & $44 \pm 5.4$ & $48.1 \pm 11$ & $84.4 \pm 28.5$ & $66.1 \pm 14.7$ & 100.0 & 0 & 0 \\
\hline
\end{tabular}

activitiesare expressed as nmol of oxidized NADH (complex I) and reduced cytochrome c (complex I+III) per min per mg of mitochondrial protein. ${ }^{b}$ Compared activitiesare displayed as the ratio of individual activities to that of citrate synthase. 'The control values are represented as the mean \pm SD of values obtained for 200 healthy persons.

\section{Mitochondrial gene sequence and copy number analysis in patients and core pedigree}

The m.3243 $A>G$ mutation in the mitochondrial tRNA ${ }^{\text {Leu }}$ gene was detected in all 
patients and their mothers. An analysis of the load of this mutation was performed in the three families by restriction fragment length polymorphism (RFLP). The mutation loads of patients 1 , 2 , and 3 were $48.3,57.2$, and $45.5 \%$, and those of their mothers were $20.5,16.4$, and $23.6 \%$, respectively, as shown in Table 2. Significant differences were observed between the patient and mother groups $(P<0.05)$. However, no correlation was found between the mutant loads of mothers and patients $(P>0.05)$. The leukocyte mtDNA copy numbers of patients and their mothers were within the control range. No statistical difference was observed between the patients and their mothers $(P>0.05)$.

\section{DISCUSSION}

Disorders in the mitochondrial respiratory chain are degenerative diseases; these show multi-system involvement in the progressive decline in the ability to meet cellular energy requirements. Deficiencies in complexes I and IV are the most frequently documented causes for mitochondrial respiratory chain disease. In contrast, deficiencies in complex III of the respiratory chain are rare, with very few patients with an isolated complex III defect being reported. The estimated incidence of complex III deficiency accounts for approximately $2 \%$ of the respiratory chain diseases in Netherlands (Mourmans et al., 1997), and 15\% of these diseases in France (Ristin et al., 1994). In a majority of the cases, disease onset is during infancy and early childhood; however, adults have also been affected in a small number of cases. A previous study of complex III deficiency in 6 patients showed that the clinical onset of the disease had occurred prior to the age of 15 months in 5 patients, and after 7 years in 1 patient (Mourmans et al., 1997). Blazquez summarized the clinical and biochemical characteristics of 14 previously reported patients with complex III deficiency, caused by mutations in the BCS1L gene. This revealed that the clinical onset was during the neonatal period or after 1 year of age in $75 \%$ and $7 \%$ of the cases, respectively. The mortality rate within 1 year of age was observed to be $62 \%$ (Blasquez et al., 2009), which indicated the earlier onset of the disease resulted in greater disease progression and a greater risk of mortality, in a majority of the cases. Among the three reported patients, one patient manifested late onset motor regression, a phenotype specific to complex III deficiency, characterized by various combinations of weakness, and mental and motor impairment (Reichmann et al., 1986; Slipetz et al., 1991). Two patients showed clinical onsets at birth.

Despite the low incidence rate, diseases resulting from defects in complex III display a wide spectrum of clinical presentation (manifestation), and predominantly affect highly oxidative tissues. Complex III deficiencies follow a progressive course, and are rarely stable. A number of patients display characteristics such as low birth weight, proximal tubulopathy, hepatopathy, and progressive neurological abnormalities. Neurological manifestations dominate these clinical symptoms. Neurological dysfunction was principally characterized by development delay, hypotonia, and encephalopathy (Blasquez et al., 2009). The major clinical symptoms associated with nervous system function exhibited by the three patients in this study (caused by lesions in the system) included motor and mental developmental delay, hypotonia, and motor regression; this was consistent with the previously reported clinical presentation of complex III deficiency. However, none of the patients displayed the presence of lesions affecting liver or renal function. This can be explained by the fact that the follow-up of patients could be short-term only, or that these patients may be affected by only nervous system dysfunction. Complex III deficiencies result in special clinical phenotypes, such as gracile syndrome (which is characterized by growth retardation, 
severe renal and liver diseases, and early death) (Visapaa et al., 2002), Bjornstad syndrome (characterized by a restricted phenotype, including hearing loss and twisted hair) (Hinson et al., 2007), Fanconi syndrome (characterized by failure of proximal renal tubular reabsorption of amino acids, glucose, and phosphate) (Morris et al., 1995), Leigh syndrome (which is characterized by the progressive decline of central nervous function, caused by focal, necrotizing lesions in the basal ganglia, diencephalon, or brainstem) (de Lonlay et al., 2001), and Leber's hereditary optical neuropathy (Brown et al., 1992). The brain MRI scans and clinical manifestations of two patients revealed the presence of focal necrotizing lesions in the basal ganglia or thalamus, compatible with the diagnosis of Leigh syndrome.

Mitochondrial complex III deficiency is caused by mutations in several different nuclear and mitochondrial genes. The m.3243 $A>G$ mutation is reportedly associated with defects in the respiratory chain complex of the cell, because of the decrease in the rate of synthesis of mitochondrial translation products (Ma et al., 2013). Several types of respiratory complex defects caused by m.3243 $A>G$ have been previously reported. Ciafaloni et al. reported that the mutation resulted in a decrease in the activities of complexes I + III, II + III, and IV in the muscles (Ciafaloni et al., 1992). In a previous study, we observed that deficiencies in complex I or IV were common; however, deficiencies in complex III were not observed in patients displaying the m.3243 A>G mutation (Ma et al., 2013). In this study, patients harboring the m.3243 A>G mutation showed the presence of complex III deficiency in the blood. Interestingly, their asymptomatic mothers harboring same mutation showed normal complex III activity. The clinical and biochemical heterogeneity may be explained by changes in the mutation load. Hammans et al reported that the m.3243 $A>G$ mutant load was significantly greater in the blood of symptomatic, rather than asymptomatic subjects, and was correlated with a clinical severity score (Huang et al., 1994). Another study reported that the median threshold for mutant load among probands was $23 \%$ in blood and $67 \%$ in muscle (Majamaa et al., 1998). In this study, the mutant loads of the three patients were 48.3, 57.2, and $45.5 \%$, while those of their asymptomatic mothers were $20.5,16.4$, and $23.6 \%$ in blood, which was in a similar range to that observed in previous studies. This finding suggests that different biochemical presentation may be associated with the importation of nucleus-encoded tRNA into mitochondria as a complement, in addition to the level of the m.3243 A>G mutation. Nuclear genes related to complex III should be detected in future studies.

Respiratory chain complex III deficiency is a relatively rare mitochondrial disease. The m.3243 $A>G$ mutation has been proven to result in mitochondrial diseases. Patients with this mutation principally displayed neurological dysfunction, such as developmental delay, hypotonia, and encephalopathy. Patient carrying the m.3243 $A>G$ mutation may biochemically express a deficiency in the mitochondrial respiratory chain complex III. High mutation load in the blood may result in the development of respiratory complex defects.

\section{Conflicts of interest}

The authors declare no conflict of interest.

\section{ACKNOWLEDGMENTS}

Research supported by grants provided by the National Nature Science Foundation of China (\#81400939), the China Postdoctoral Science Foundation (\#2013M532107), and the 
Scientific Research Guidance Project of Qinghai Health and Family Planning (\#2014QHFP0928).

\section{REFERENCES}

Birch-Machin MA and Turnbull DM (2001). Assaying mitochondrial respiratory complex activity in mitochondria isolated from human cells and tissues. Methods Cell Biol. 65: 97-117.

Blazquez A, Gil-Borlado MC, Moran M, Verdu A, et al. (2009). Infantile mitochondrial encephalomyopathy with unusual phenotype caused by a novel BCS1L mutation in an isolated complex III-deficient patient. Neuromuscul. Disord. 19: 143-146.

Brown MD, Voljavec AS, Lott MT, Torroni A, et al. (1992). Mitochondrial DNA complex I and III mutations associated with Leber's hereditary optic neuropathy. Genetics 130: 163-173.

Ciafaloni E, Ricci E, Shanske S, Moraes CT, et al. (1992). MELAS: clinical features, biochemistry, and molecular genetics. Ann. Neurol. 31: 391-398.

de Lonlay P, Valnot I, Barrientos A, Gorbatyuk M, et al. (2001). A mutant mitochondrial respiratory chain assembly protein causes complex III deficiency in patients with tubulopathy, encephalopathy and liver failure. Nat. Genet. 29: 57-60.

Guzy RD, Hoyos B, Robin E, Chen H, et al. (2005). Mitochondrial complex III is required for hypoxia-induced ROS production and cellular oxygen sensing. Cell Metab. 1: 401-408.

Hammans SR, Sweeney MG, Hanna MG, Brockington M, et al. (1995). The mitochondrial DNA transfer RNALeu(UUR) A-$>\mathrm{G}(3243)$ mutation. A clinical and genetic study. Brain 118: 721-734.

Hinson JT, Fantin VR, Schonberger J, Breivik N, et al. (2007). Missense mutations in the BCS1L gene as a cause of the Bjornstad syndrome. N. Engl. J. Med. 356: 809-819.

Huang CC, Chen RS, Chen CM, Wang HS, et al. (1994). MELAS syndrome with mitochondrial tRNA(Leu(UUR)) gene mutation in a Chinese family. J. Neurol. Neurosurg. Psychiatry 57: 586-589.

Iwata S, Lee JW, Okada K, Lee JK, et al. (1998). Complete structure of the 11-subunit bovine mitochondrial cytochrome bc1 complex. Science 281: 64-71.

Ma YY, Zhang XL, Wu TF, Liu YP, et al. (2011). Analysis of the mitochondrial complex I-V enzyme activities of peripheral leukocytes in oxidative phosphorylation disorders. J. Child Neurol. 26: 974-979.

Ma YY, Wu TF, Liu YP, Wang Q, et al. (2013). Heterogeneity of six children and their mothers with mitochondrial DNA 3243 A>G mutation. Mitochondrial DNA 24: 297-302.

Majamaa K, Moilanen JS, Uimonen S, Remes AM, et al. (1998). Epidemiology of A3243G, the mutation for mitochondrial encephalomyopathy, lactic acidosis, and strokelike episodes: prevalence of the mutation in an adult population. Am. J. Hum. Genet. 63: 447-454.

Miller SA, Dykes DD and Polesky HF (1988). A simple salting out procedure for extracting DNA from human nucleated cells. Nucleic Acids Res. 3: 1215.

Morris AA, Taylor RW, Birch-Machin MA, Jackson MJ, et al. (1995). Neonatal Fanconi syndrome due to deficiency of complex III of the respiratory chain. Pediatr. Nephrol. 9: 407-411.

Mourmans J, Wendel U, Bentlage HA, Trijbels JM, et al. (1997). Clinical heterogeneity in respiratory chain complex III deficiency in childhood. J. Neurol. Sci. 149: 111-117.

Reichmann H, Rohkamm R, Zeviani M, Servidei S, et al. (1986). Mitochondrial myopathy due to complex III deficiency with normal reducible cytochrome b concentration. Arch. Neurol. 43: 957-961.

Rustin P, Chretien D, Bourgeron T, Gerard B, et al. (1994). Biochemical and molecular investigations in respiratory chain deficiencies. Clin. Chim. Acta 228: 35-51.

Schaefer AM, Taylor RW, Turnbull DM and Chinnery PF (2004). The epidemiology of mitochondrial disorders - past, present and future. Biochim. Biophys. Acta 1659: 115-120.

Slipetz DM, Aprille JR, Goodyer PR and Rozen R (1991). Deficiency of complex III of the mitochondrial respiratory chain in a patient with facioscapulohumeral disease. Am. J. Hum. Genet. 48: 502-510.

Visapaa I, Fellman V, Vesa J, Dasvarma A, et al. (2002). GRACILE syndrome, a lethal metabolic disorder with iron overload, is caused by a point mutation in BCS1L. Am. J. Hum. Genet. 71: 863-876.

Xing J, Chen M, Wood CG, Lin J, et al. (2008). Mitochondrial DNA content: its genetic heritability and association with renal cell carcinoma. J. Natl. Cancer Inst. 100: 1104-1112. 